\title{
DESIGN OF V-BAND SUBSTRATE INTEgRATED WAVEguide POWER Divider, CirCUlATOR AND COUPLER
}

\author{
Bouchra Rahali ${ }^{1}$ and Mohammed Feham ${ }^{2}$ \\ ${ }^{1}$ STIC Laboratory University of Tlemcen, Algeria \\ b_rahali@hotmail.fr \\ ${ }^{2}$ STIC Laboratory University of Tlemcen, Algeria \\ m. fehamemail.univ-tlemcen.dz
}

\begin{abstract}
Recently there is growing interest in a new technology, substrate integrated waveguide (SIW), it has been applied successfully to the conception of planar compact components for the microwave and millimeter waves applications. In this study, a V-band substrate integrated waveguide power divider, circulator and coupler are conceived and optimized by Ansoft HFSS code. Thus, through this modeling, design considerations and results are discussed and presented. Attractive features including compact size and planar form make these devices structure easily integrated in planar circuits.
\end{abstract}

\section{KEYWORDS}

Rectangular Waveguide, Microwave Components, SIW, Power Divide, Circulator, Coupler, HFSS.

\section{INTRODUCTION}

To obtain the benefits of rectangular waveguides while remaining in planar profiles, the SIW technology [1] [2] is interesting. The rectangular waveguide in the technology SIW (RSIW) has some interesting characteristics in terms of easy integration while offering components to a high quality factor. A large range of SIW components such as bends [3], filters [4], couplers [5], duplexers [6], sixports [7], circulators [8] and phase shifters [9] has been proposed and studied at low cost, high quality, relatively high power and integration with other microwave components in the same dielectric substrate [1] [2].The (RSIW) (Figure 1) is fabricated using two rows of periodic metallic posts witch connect two higher and lower planes mass of dielectric substrate effectively the geometry and the distribution of the electric field in (RSIW), illustrated in Figure 2 and 3 are similar to those of the equivalent rectangular wave guide [2] [3]. In this paper, V-band RSIW components are proposed and optimized. They are building blocks of many microwave and millimeter-wave integrated circuits and telecommunication systems.

\section{FUNDAMENTAL RSIW CHARACTERISTICS}

From a dielectric substrate between two metal planes, two rows of holes are drilled and metalized, making contact between the two metal planes of the substrate, allowing propagation of all modes $\mathrm{TE}_{\mathrm{n} 0}$ [5]. The diameter $\mathrm{d}$ of holes stems, $\mathrm{p}$ the spacing between the holes and $\mathrm{W}_{\mathrm{SIW}}$ spacing between the two rows of holes are physical parameters necessary for designing (RSIW) (Figure 1). Indeed its electrical behavior is similar to that of a conventional rectangular waveguide filled 
with dielectric of width $\mathrm{W}_{\text {eq }}$ [4]. The current lines along the side walls of the RSIW is vertical, the fundamental mode $\mathrm{TE}_{10}$ can propagate efficiently.

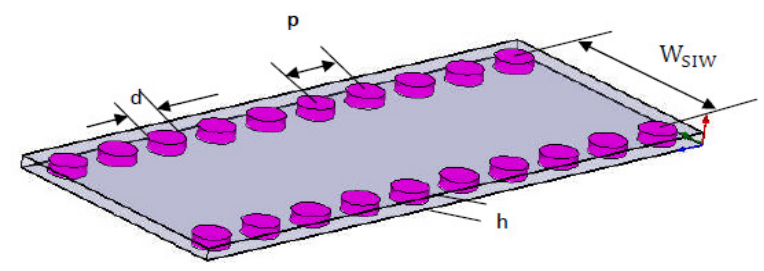

Figure 1. Rectangular wave guide integrated into a substrate RSIW

Based on the work [2], empirical equations were derived for determining the width of the equivalent rectangular wave guide, giving the same characteristics of the fundamental mode propagating in the RSIW (Figure 2) having the same height and the same dielectric.

$$
\begin{aligned}
& \mathrm{W}_{\text {eq }}=\mathrm{W}_{\text {SIW }}-\frac{\mathrm{d}^{2}}{0.95 \mathrm{p}} \\
& \mathrm{p}<\frac{\lambda_{0}}{2} \sqrt{\varepsilon_{\mathrm{r}}} \\
& \mathrm{p}<4 d
\end{aligned}
$$

$\lambda_{0}=\frac{\mathrm{c}}{\mathrm{f}}, \lambda_{0}$ is the space wavelength.

The period $\mathrm{p}$ should be kept low to reduce leakage losses between adjacent cylinders. The choice of subject is also the problem of losses.

We analyzed, the RSIW designed in V-band [50-75] GHz from a conventional waveguide [10] whose characteristic parameters are described in Table 1. Following the same approach, cited above [2], we deduce the parameters of the RSIW and the equivalent waveguide (Figure 2) Table 1.

Table 1

\begin{tabular}{|l|l|l|}
\hline \multicolumn{1}{|c|}{ Classic wave guide } & \multicolumn{1}{|c|}{ Equivalent wave guide } & \multicolumn{1}{c|}{ RSIW } \\
\hline $\mathrm{WR} 15, \mathrm{a}=3.759 \mathrm{~mm}$, & $\mathrm{h}=0.15 \mathrm{~mm}, \varepsilon_{\mathrm{r}}=3.15$ & $\mathrm{~h}=0.15 \mathrm{~mm}, \varepsilon_{\mathrm{r}}=3.15$, \\
$\mathrm{b}=1.88 \mathrm{~mm}, \varepsilon_{\mathrm{r}}=1$ & $\mathrm{~W}_{\mathrm{eq}}=2.12 \mathrm{~mm}$ & $\begin{array}{l}\mathrm{d}=0.2 \mathrm{~mm}, \mathrm{p}=0.4 \mathrm{~mm}, \\
\mathrm{~W}_{\mathrm{SIW}}=2.24 \mathrm{~mm}\end{array}$ \\
\hline
\end{tabular}

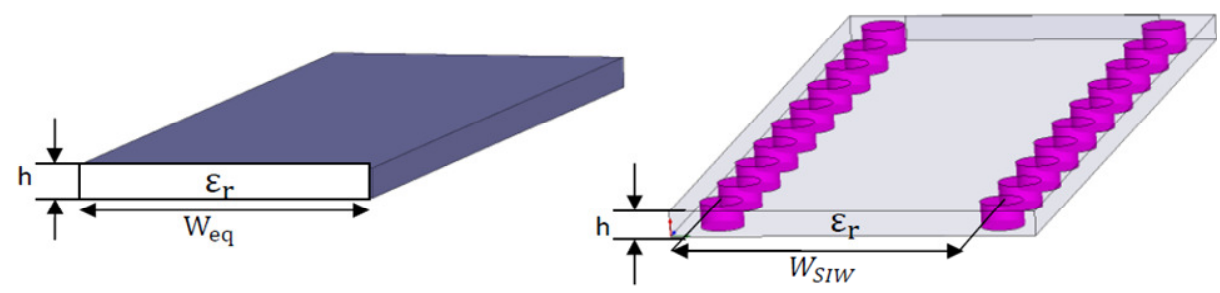

Figure 2. Equivalent rectangular waveguide and RSIW 
In this study, we have analyzed many structures with HFSS tool [11] based on the finite element method (FEM). It should be noted that the formulas given by equations (1), (2) and (3) are commonly used to obtain initial values $\mathrm{W}_{\mathrm{SIW}}$, optimized later by HFSS. It has allowed the layout of the cartography of the electromagnetic field of the $\mathrm{TE}_{10}$ mode and scatter diagram. Figure 3 shows the similarity of the electromagnetic field distribution of $\mathrm{TE}_{10}$ mode guided in the guide RSIW and its equivalent in waveguide technology.

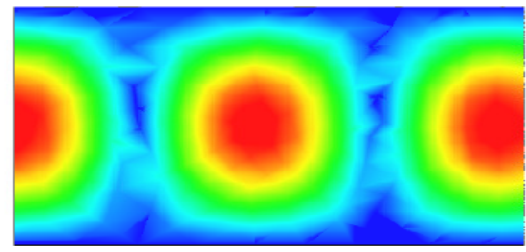

(a)

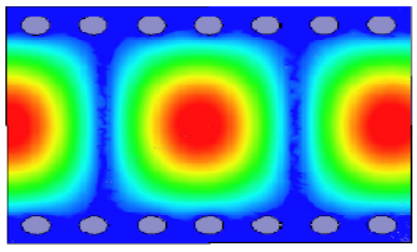

(b)

Figure 3. Electric field distribution of the $\mathrm{TE}_{10}$ mode in the equivalent rectangular waveguide (a) and RSIW (b) at the frequency $\mathrm{f}=60 \mathrm{GHz}$

Figure 4 also shows the consistency of the dispersion characteristics between these two equivalent wave guides. It should be noted that this similarity propagation is valid for all modes $\mathrm{TE}_{\mathrm{n} 0}$.

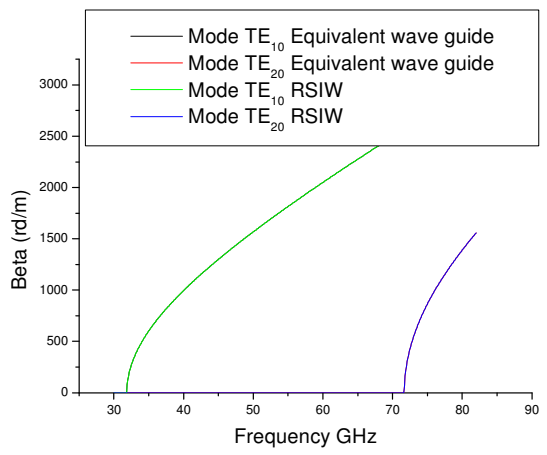

Figure 4. Dispersion characteristics

\section{RSIW-MICROSTRIP TAPERED TRANSITION}

The microstrip transition taper [12] employed to interconnect RSIW to the planar transmission lines is used in this paper. There is a tapered section which is used to match the impedance between a $50 \Omega$ microstrip line and the RSIW. The $50 \Omega$ microstrip line, in which the dominant mode is quasi-TEM, can excite well the dominant mode $\mathrm{TE}_{10}$ of the RSIW, as their electric field distributions are approximate in the profile of the structure.

Initial parameters $\mathrm{W}_{T}$ and $\mathrm{L}_{\mathrm{T}}$ are determined from several formulas given [13], following by an optimization using the HFSS [11] Table 2. Figure 5 shows the proposed configuration of two back-to-back transitions of microstrip line to RSIW. Figures 6 and 7 show the results of the RSIW analysis without transition and with a coplanar taper of dimensions $\mathrm{L}_{\mathrm{T}}$ and $\mathrm{W}_{\mathrm{T}}$.

Table 2

\begin{tabular}{|l|l|}
\hline $\mathrm{L}_{\mathrm{T}}$ & $1.57 \mathrm{~mm}$ \\
\hline $\mathrm{W}_{\mathrm{T}}$ & $0.77 \mathrm{~mm}$ \\
\hline $\mathrm{W}_{\mathrm{mst}}$ & $0.37 \mathrm{~mm}$ \\
\hline $\mathrm{L}$ & $2.8 \mathrm{~mm}$ \\
\hline
\end{tabular}




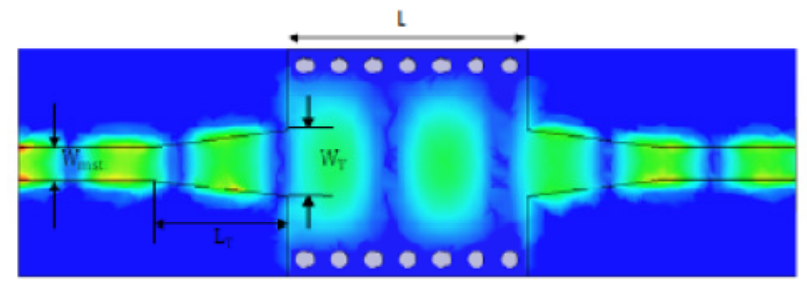

Figure 5. Electric field distribution of $\mathrm{TE}_{10}$ mode at $\mathrm{f}=60 \mathrm{GHz}$ in the matched RSIW

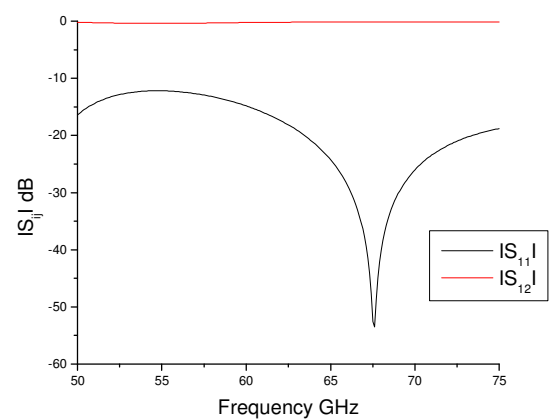

Figure 6. Transmission coefficients $\mathrm{S}_{21}$ and reflection $\mathrm{S}_{11}$ of the RSIW

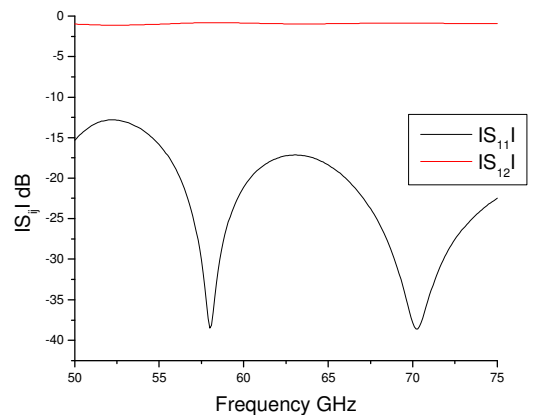

Figure 7. Transmission coefficients $S_{21}$ and reflection $S_{11}$ of the matched RSIW with taper

The results illustrated in figure 7, indicate that the reflection coefficient $S_{11}$ remains below-15 dB over $31.51 \%$ of the frequency band and the transmission coefficient $S_{21}$ is around $-0.88 \mathrm{~dB}$ across the entire band. This concept [13] allows the design of a completely integrated planar circuit of microstrip and waveguide on the same substrate without any mechanical assembly [14] [15].

\section{Design OF RSIW Passive DeVICES}

\subsection{SIW Power Divider}

The power dividers [16] are passive microwave devices. There are mainly two types $\mathrm{T}$ and $\mathrm{Y}$ [14] [16], which are commonly used to deliver copies of a signal in a system. This application focuses on the three ports power dividers with equal power division ratio where the half power $(-3 \mathrm{~dB})$ of an input signal is provided to each of the two output ports. The $\mathrm{S}$ matrix for a three ports network, is shown in equation (4)

$$
[S]=\left[\begin{array}{lll}
S_{11} & S_{12} & S_{13} \\
S_{21} & S_{22} & S_{23} \\
S_{31} & S_{32} & S_{33}
\end{array}\right]
$$

The analyzed power divider (Figure 8), designed in the V- band [50-75] GHz, is based on three identical RSIW with the characteristic parameters reported in Table 1 and 2. Then Three RSIW of length $\mathrm{L}=2.8 \mathrm{~mm}$ are connected to form a $\mathrm{T}$. An inductive metal cylinder of radius $\mathrm{r}$ and position $\mathrm{xp}$ is added to this junction in order to minimize reflection losses at the input port. To achieve this objective, an optimization of the radius $r$ and the position $x p$ of this disruptive element is required. For this purpose, it is generally useful to fix the radius $r$ to the corresponding available practical value of diameter drills, and then change xp to reduce the reflection losses. This will be repeated until reflection losses, below $-15 \mathrm{~dB}$, reach acceptable limit. Then, a microstrip transition is added to each port so that we can integrate this component directly into a microstrip circuit. The input wave (port 1) is divided into two parts which output to port 2 and port 3 , respectively. 


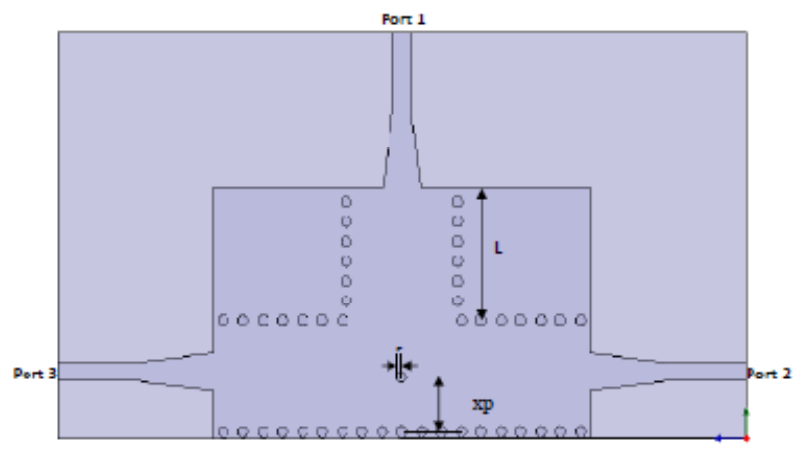

Figure 8. RSIW power divider

Figures 9 and 10 illustrate the distribution of the electric field of the $\mathrm{TE}_{10}$ mode in the band [50-75] $\mathrm{GHz}$ and the transmission coefficients $S_{21}, S_{31}$ and the reflection coefficient $S_{11}$, respectively. The figure 10 indicates that $S_{11}$ is less than $-15 \mathrm{~dB}$ between $50 \mathrm{GHz}$ and $61 \mathrm{GHz}$, which is more than $19.82 \%$ of the bandwidth. The optimal values of the inductive cylinder are $r=0.1 \mathrm{~mm}$, $x p=1.02 \mathrm{~mm}$. Transmission coefficients $S_{21}$ and $S_{31}$ fluctuate between $-3.65 \mathrm{~dB}$ and $-3.76 \mathrm{~dB}$, being very acceptable levels.

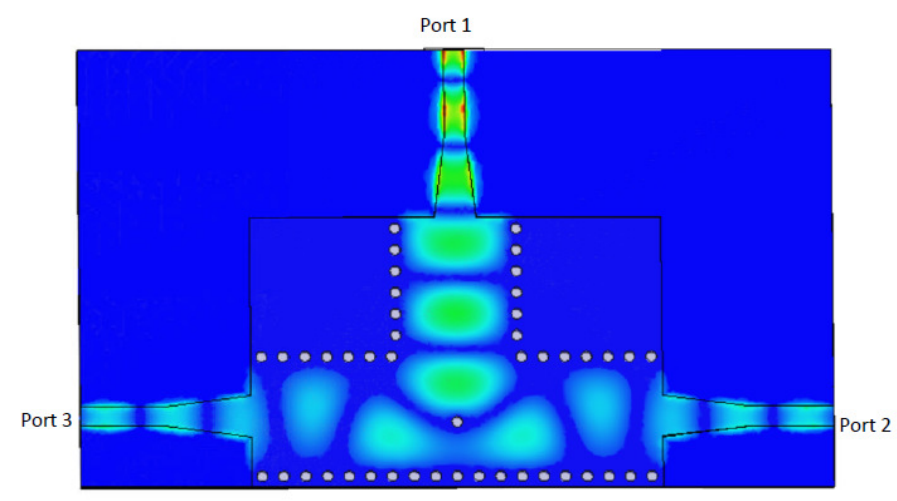

Figure 9. Electric field distribution of the $\mathrm{TE}_{10}$ mode at $\mathrm{f}=55 \mathrm{GHz}$ in the RSIW power divider with inductive cylinder

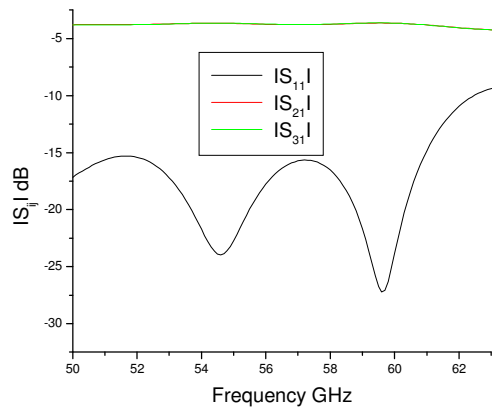

Figure 10. Parameters $S_{\mathrm{ij}}$ in the RSIW power divider with inductive cylinder

\subsection{SIW Circulator}

Protection microwave sources are a very common and recommended to increase the reliability of emitters practice. Several types of solutions are used to provide this kind of function. However, in high power, the circulator waveguide technology [8] is still the best solution. Its topology of a 
hexapole (Figure 11) having three access separate of $120^{\circ}$ from each other, around a central body of ferrite (nickel materials and lithium ferrite) [8] [9], to which is applied a vertical magnetic field conferring to the circulator the property of non-reciprocity. Indeed, when a transverse magnetic field is applied, it creates in its central part an internal field such that incoming wave from port 1,2 or 3 cannot out by the access 2, 3 or 1, respectively.

The two important variables for a circulator are insertion losses to be as low as possible $(<1 \mathrm{~dB})$ and good isolation $(-30 \mathrm{~dB})$. The ideal circulator is a suitable hexapole that would be able to direct the energy to the next access, the third being isolated. Its ideal matrix [S] would be as follows:

$$
[S]=\left[\begin{array}{ccc}
0 & 0 & e^{j \varphi} \\
e^{j \varphi} & 0 & 0 \\
0 & e^{j \varphi} & 0
\end{array}\right]
$$

Where $\varphi$ is the phase shift associated with transmission of the access signal to the next access. The non-symmetry of the matrix reflects clearly the non-reciprocity of the component. This nonreciprocity is the whole point of the device and explains that this function can be used in many applications in telecommunications. This circulator was designed by using cylindrical metal rods, with the same parameters presented in Tables 1 and 2, but $\mathrm{L}=2.8 \mathrm{~mm}$.

In this paper the saturation magnetization of ferrite material is [9] $4 \pi \mathrm{Ms}=5000$ Gauss. Its relative dielectric constant is 13.7 and a radius $\mathrm{R}_{\mathrm{f}}$ calculated by [8].

$$
\mathrm{R}_{\mathrm{f}}=\frac{1.84 \mathrm{c}}{\omega_{0} \sqrt{\varepsilon_{\mathrm{f}}}}
$$

Where $\mathrm{c}$ and $\omega_{0}$ are respectively the velocity of light in the free space and the operation frequency [8]. The ferrite height is equal to the RSIW thickness, $\mathrm{R}_{\mathrm{f}}=0.5 \mathrm{~mm} \mathrm{~h}_{\mathrm{f}}=0.15 \mathrm{~mm}$.

The RSIW circulator, presented in figure 11, has been simulated by HFSS [11]. Figure 12 illustrates the distribution of the electric field of the $\mathrm{TE}_{10}$ mode in the band [50-75] GHz. The frequency response of this SIW circulator, the transmission coefficient $S_{21}$, the reflection coefficient $S_{11}$ and the isolation coefficient $S_{31}$ are reported through the figure 13. The analysis of these results shows that the reflection losses $S_{11}$ below $-15 \mathrm{~dB}$ occupy more than $6 \%$ of the bandwidth, the insertion loss $S_{21}$ is about $-0.8 \mathrm{~dB}$, while the maximum of the isolation $\mathrm{S}_{31}$ is $-36.32 \mathrm{~dB}$. At frequency of $55 \mathrm{GHz}$, the two figures 12 and 13 confirm traffic property of this device [14].

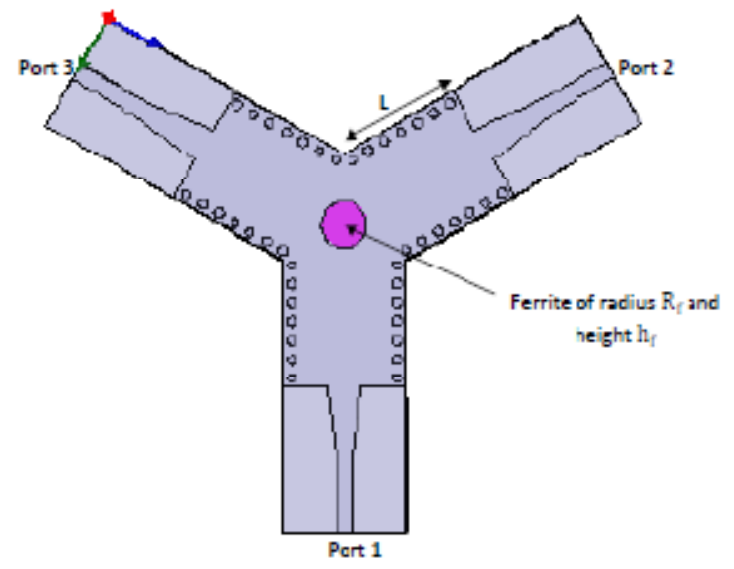

Figure 11. RSIW circulator 


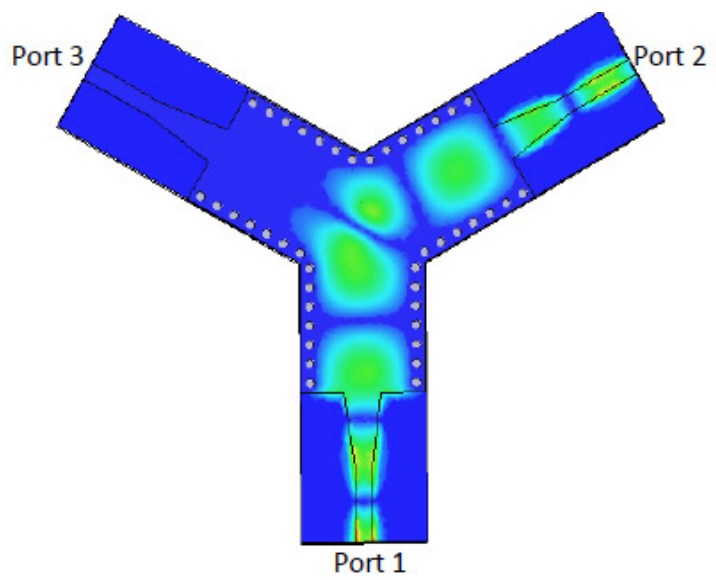

Figure 12. Electric field distribution of the $\mathrm{TE}_{10}$ mode of the RSIW circulator at $\mathrm{f}=55 \mathrm{GHz}$

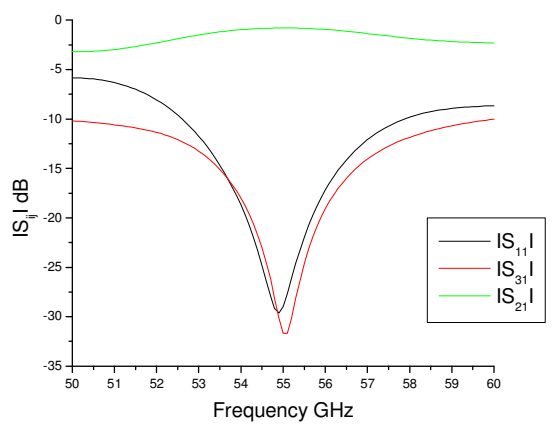

Figure 13. Parameters $S_{i j}$ of RSIW circulator

\subsection{SIW Coupler}

The development of modern communication systems, in microwave and millimeter waves domain, requires high quality and high density integration circuits. The size and cost are two essential requirements of these systems. This has stimulated the rapid development of many passive compact [14] [15] and low cost components. Couplers [5] which have been widely used as key components in many systems have been intensively studied for decades. The directional couplers are passive devices used for routing, dividing and combining the signals in the microwave system. In the antenna beam-forming networks, the directional coupler is generally an important element in power dividing/combining networks, so great interest and effort have been directed to the development of different types of directional couplers [17]. The RSIW directional couplers are extensively investigated. Coupler's configuration is illustrated in figure 14. The RSIW directional coupler $(-3 \mathrm{~dB})$ is realized by two RSIW with a common wall on which an aperture is utilized to realize the coupling between these two guides. The geometry of the coupler is determined [15] based on an even/odd mode analysis, where the even mode is the $\mathrm{TE}_{10}$ and the odd mode is the $\mathrm{TE}_{20}$. The phase difference $\Delta \varphi$ between the two modes is expressed by

$$
\Delta \varphi=\left(\beta_{1}-\beta_{2}\right) \mathrm{W}_{\mathrm{ap}}
$$

Where $\beta_{1}$ and $\beta_{2}$ are the propagation constants of the $\mathrm{TE}_{10}$ and $\mathrm{TE}_{20}$ modes, respectively. In the band of operation, the condition $\Delta \varphi=\pi / 2$ needs to be satisfied. Port 1 is the input port, port 2 is named as the through port, port 3 is the coupling port and port 4 is designed as the isolated port. 
The tapered transition between the $50 \Omega$ microstrip line and the RSIW is added to each port so that we can integrate this component directly into a microstrip circuit. The matrix [S] of a symmetric coupler $(-3 \mathrm{~dB})$, adapted to its access, is given by equation (8).

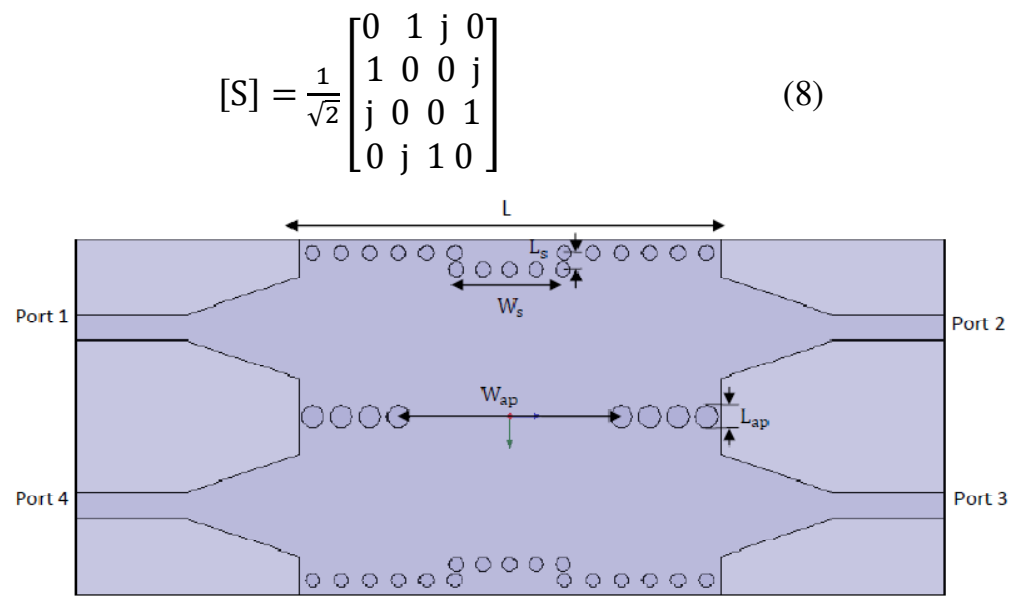

Figure 14. RSIW coupler

The coupler parameters are finely tuned using three-dimensional (3D) electromagnetic (EM) simulation under HFSS [11] to achieve wide-band performance. The final dimensions of the SIW coupler are presented in Table 3.

Table 3

\begin{tabular}{|c|l|}
\hline $\mathrm{L}$ & $5.93 \mathrm{~mm}$ \\
\hline $\mathrm{W}_{\mathrm{s}}$ & $1.498 \mathrm{~mm}$ \\
\hline $\mathrm{L}_{\mathrm{s}}$ & $0.227 \mathrm{~mm}$ \\
\hline $\mathrm{W}_{\mathrm{ap}}$ & $3.138 \mathrm{~mm}$ \\
\hline $\mathrm{L}_{\mathrm{ap}}$ & $0.313 \mathrm{~mm}$ \\
\hline
\end{tabular}

Figures 15 and 16 present electric field distribution of the $\mathrm{TE}_{10}$ mode in the V-band and the reflection coefficients $S_{11}$, transmission coefficients $S_{21}$, coupling and isolation coefficients $S_{31}, S_{41}$. The results of this analysis show clearly the directional coupler character in the band [50-75] GHz, where we have the levels of reflection and isolation below $-15 \mathrm{~dB}$ in more than $26.43 \%$ of the bandwidth, and the insertion losses $S_{21}$ and coupling $S_{31}$ fluctuate between $-3.59 \mathrm{~dB} \pm 0.5 \mathrm{~dB}$. These simulation results prove the good performance of this integrated structure.

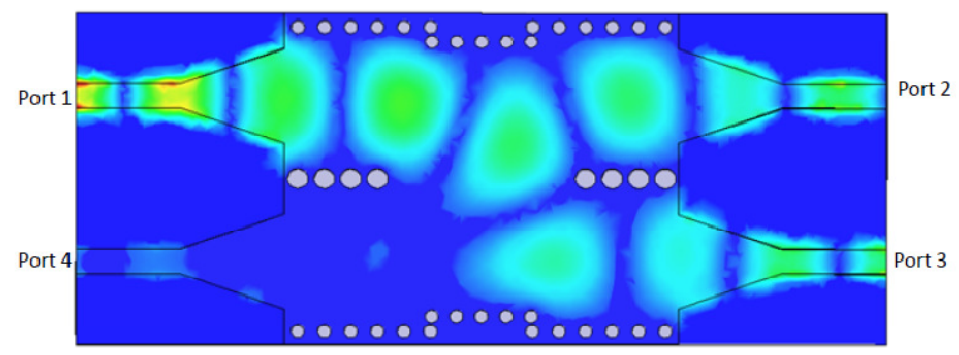

Figure 15. Electric field distribution of $\mathrm{TE}_{10}$ mode for RSIW coupler at $\mathrm{f}=60 \mathrm{GHz}$ 


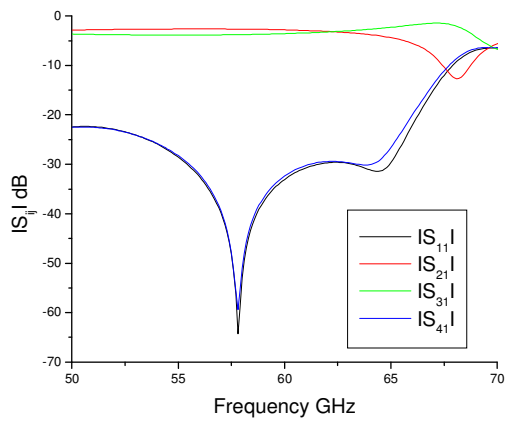

Figure 16. Frequency response of the RSIW directional coupler

\section{CONCLUSIONS}

In this paper, we have investigated a V-band substrate integrated waveguide passive components. We have presented a simple and fast method to design three passive components based on RSIW using Ansoft HFSS code. Firstly, the RSIW three ports power dividers with equal power division ratios where half the power of an input signal is provided to each of the two output ports. Secondly, the V-band RSIW circulator, suitable for millimeter wave applications, has been designed; it can be integrated on the same substrate with microstrip and other planar circuit. The HFSS code has also been applied to the analysis of the V-band coupler which is composed of two matched RSIW with a common wall on which an aperture is used to realize the coupling. The developed RSIW power dividers, circulator and coupler can serve as building blocks in the design of complex microwave and millimeter wave circuits and systems. The simulation results have shown the good performance of these integrated structures.

\section{REFERENCES}

[1] D. Deslandes and K. Wu, (2002) "Design Consideration and Performance Analysis of Substrate Integrated Waveguide Components,” Europ. Microw. Conf., pp.1-4, Oct.

[2] Y. Cassivi, L. Perregrini, P. Arcioni, M. Bressan, K. Wu, G. Conciauro, (2002) "Dispersion Characteristics of Substrate Integrated Rectangular Waveguide" IEEE Microw. Wireless Comp. Lett., Vol. 12, No. 9, pp. 333-335.

[3] Rahali Bochra, Feham Mohammed, Junwu TAO, (2011) "Design of optimal chamfered bends in Rectangular Substrate Integrated Waveguide", IJCSI, International Journal of Computer Science Issues, Vol. 8, Issue 4, No 2.

[4] A. Adabi and M. Tayarani , (2008) "Substrate Integration of Dual Inductive Post Waveguide Filter", Electrical Engineering Department IUST University Narmak, Tehran, Iran Progress In Electromagnetics Research B, Vol. 7, 321-329.

[5] Tan Ke-jun, Luan Xiu-zhen,(2009) "Compact Directional Coupler Based on Substrate integrated Waveguide", IEEE Microwave and Wireless Components Letters .

[6] Z. C. Hao, W. Hong, J. X. Chen, X. P. Chen and K. Wu, (2005) "Planar diplexer for microwave integrated circuits“, IEE Proc.-Microw. Antennas Propag., Vol. 152, No. 6.

[7] Xinyu Xu, Renato G. Bosisioand and Ke Wu, (2005) "A New Six-Port Junction Based on Substrate Integrated Waveguide Technology", IEEE Transactions on Microwave Theory and Techniques, Vol. 53, No. 7.

[8] Wenquan Che, Xiao Jing Ji,Edward K. N. Yung, (2007) "Miniaturized Planar Ferrite Junction Circulator in the Form of Substrate-Integrated Waveguide", International Journal of RF and Microwave Computer-Aided Engineering.

[9] Y. J. Ban , (2010) "Tunable Ferrite Phase Shifters Using Substrate Integrated Waveguide Technique" Département de Génie Electrique Ecole Polytechnique de Montréal.

[10] Rahali Bouchra , (2013) "Contribution à la Modélisation Electromagnétique des structures Complexes Hyperfréquences en Technologie SIW”, Thèse de doctorat, Département de Génie Electrique et Electronique, Faculté de Technologie, Université Abou Bekr Belkaid de Tlemcen Algérie. 
[11] User's guide - High Frequency Structure Simulator (HFSS), v11.0 Ansoft Corporation.

[12] Dominic Deslandes and Ke Wu, (2001) "Integrated Micro strip and Rectangular Waveguide in Planar Form", Microwave and Wireless Components Letters, IEEE, pp.68-70.

[13] Dominic Deslandes , (2010) "Design Equations for Tapered Microstrip-to-Substrate Integrated Waveguide Transitions”, Microwave Symposium Digest, IEEE MTT-S International, pp. 704-707.

[14] Nathan Alexander Smith, (2010) "Substrate Integrated Waveguide Circuits and Systems", Thesis for the degree of Master of Engineering, Department of Electrical \& Computer Engineering McGill University Montréal, Québec, Canada.

[15] Teis Coenen, (2010) "Analysis and Design of Post Wall Waveguides for Applications in SIW” , PhD Thesis, the Telecommunication Engineering group of the Faculty of Electrical Engineering, Mathematics and Computer Science of the University of Twente Enschede, The Netherlands.

[16] Simon Germain, Dominic Deslandes , Ke Wu ,(2003) "Development of Substrate Integrated Waveguide Power Dividers", Electrical and Computer Engineering, IEEE, Vol.3, pp.1921-1924.

[17] Tarik Djerafi, (2011) "Etude et Réalisation de Matrices à Commutation de Faisceaux en Technologie Guide D'ondes Integré au Substrat", These de ph.D Genie Electrique, Département de Génie Electrique Ecole Polytechnique de Montreal. 